\title{
Attosecond Angle-Resolved Photoelectron Spectroscopy
}

\author{
S. A. Aseyev, Y. Ni, L. J. Frasinski, ${ }^{*}$ H. G. Muller, and M. J. J. Vrakking \\ FOM-Institute AMOLF, Kruislaan 407, 1098 SJ Amsterdam, The Netherlands
}

(Received 1 May 2003; published 26 November 2003)

\begin{abstract}
We report experiments on the characterization of a train of attosecond pulses obtained by highharmonic generation, using mixed-color (XUV + IR) atomic two-photon ionization and electron detection on a velocity map imaging detector. We demonstrate that the relative phase of the harmonics is encoded both in the photoelectron yield and the angular distribution as a function of XUV-IR time delay, thus making the technique suitable for the detection of single attosecond pulses. The timing of the attosecond pulse with respect to the field oscillation of the driving laser critically depends on the target gas used to generate the harmonics.
\end{abstract}

DOI: 10.1103/PhysRevLett.91.223902

The development of ultrashort laser pulses has led to significant advances in physics, chemistry, and biology. With femtosecond lasers the elementary motions of atoms and molecules can be observed [1], leading, for example, to a better understanding of complex biological systems [2]. Although electron dynamics often plays a crucial role in these systems, experimental studies have thus far been mostly limited to investigating the "slow" particles (i.e., atoms) that move under the influence of the intramolecular potentials. In order to investigate electron dynamics in real time, pulse durations have to enter the subfemtosecond (also known as attosecond, 1 as $=1 \times$ $10^{-18} \mathrm{~s}$ ) domain [3]. Important advances have been made in the experimental realization and detection of attosecond laser pulses [4-6] formed through the generation of high-order harmonics of femtosecond infrared (IR) laser pulses in noble gas atoms. In that process, attosecond time structure results from phase locking of a series of discrete harmonics $[5,7,8]$ or a sufficiently broad segment of the harmonics continuum [6]. In this work, the time structure of such attosecond pulses is characterized from the angular distribution of the photoelectrons they produce in atomic mixed-color two-photon ionization.

Many problems still have to be solved before well characterized and controlled attosecond pulses can be used as a routine tool for time-resolved spectroscopy $[3,9]$. When many-cycle IR pulses are used for the harmonic generation, the attosecond pulses appear in a pulse train, which is incompatible with most pump-probe experiments. Therefore the ultimate goal is to produce a single attosecond pulse. Another problem is the characterization of attosecond pulses. Full characterization can be done only through a nonlinear process, since linear processes do not mix different frequency components $A_{q} \cos \left(\omega_{q} t+\varphi_{q}\right)$ and thus are insensitive to their relative phase $\varphi_{q}-\varphi_{q^{\prime}}$. Knowledge of the latter is essential and (together with the easily measurable amplitudes $A_{q}$ ) sufficient for determining a complete field reconstruction $\sum A_{q} \exp \left[i\left(w_{q} t+\varphi_{q}\right)\right]$ except for an overall phase.

Thus far, mixed-color multiphoton ionization involving one extreme ultraviolet (XUV) and one or more
PACS numbers: 42.65.Ky, 32.80.Rm, 42.65.Re

fundamental photons has been the only process delivering enough signal to allow such measurements $[5,6]$. In the low-intensity limit, the amplitude of this process is proportional to the amplitudes of the involved fields, phase factors included. Ionization by absorption of a harmonic $q$ and an IR photon interferes with ionization by the nexthigher harmonic $q+2$ accompanied by (stimulated) IR emission. If the fields are represented as $\cos (\omega t+\varphi)=$ $\frac{1}{2}\left\{e^{i(\omega t+\varphi)}+e^{-i(\omega t+\varphi)}\right\}$, the positive and negative frequency components (responsible for absorption and emission, respectively) have opposite phases. The phase difference of the interfering pathways then contains a phase $\varphi_{q}-\varphi_{q-2}+2 \varphi_{\mathrm{IR}}$, so that mapping the interference as a function of $\varphi_{\text {IR }}$ (through retarding the IR beam) reveals the sought relative phase $\varphi_{q}-\varphi_{q-2}$ in the offset of the fringe maxima from the zero-retardation point.

Previously, in an experiment by Paul et al. [5], transitions involving high-order harmonics and the fundamental IR laser were observed in a focused geometry using a time-of-flight electron spectrometer, where the photoelectron signal was integrated over all ejection angles. This method would run into trouble when characterizing single attosecond pulses or very short trains of such pulses, where photoelectrons produced by single- and two-color (XUV + IR) multiphoton ionization have overlapping energy distributions. Therefore it is essential to find alternative ways to distinguish single-photon and two-photon events. In this paper, we demonstrate that the angular distribution of the photoelectrons can be used to this end.

Our experiment uses an in-line geometry, where the XUV and IR beams are not refocused, thus creating optimally "clean conditions" for the determination of the relative phases of the high-order harmonics (Fig. 1). Photoionization of the target gas by harmonics is accompanied by absorption and emission of IR photons. In the photoelectron spectrum, this results in the appearance of sideband peaks centered between the single-photon XUV ionization signals [11]. For low enough IR intensity, only two adjacent harmonics contribute to each sideband, making observation of the sidebands as a function of 


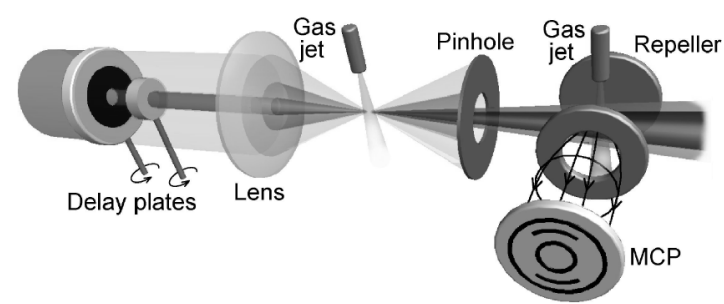

FIG. 1. Overview of the experimental scheme. The output of a $50 \mathrm{~Hz}$ Ti:sapphire laser system (delivering near-transform limited $70 \mathrm{fs}, 10 \mathrm{~mJ}$ laser pulses at $800 \mathrm{~nm}$ ) is split into an annular outer beam (adjustable up to a diameter $\varnothing_{\text {out }}=$ $20 \mathrm{~mm})$ and an inner beamlet $(\varnothing=3 \mathrm{~mm})$, which are delayed with respect to each other by passage through two pieces of glass cut from the same antireflection-coated plate. An $f=$ $2 \mathrm{~m}$ lens focuses the copropagating IR beams into a rare gas jet, where the annular beam generates harmonics. A masking plate with a $\varnothing=0.5 \mathrm{~mm}$ pinhole blocks the annular part of the IR beam $20 \mathrm{~cm}$ behind the focus, and transmits the inner IR beam and the harmonics into a spectrometer chamber. There the lasers cross an atomic beam and the resulting photoelectrons are detected using velocity imaging, which projects the electrons with the aid of electrostatic fields onto a position-sensitive channel-plate detector. The $3 \mathrm{D}$ velocity distribution is recovered from the measured projection by applying an iterative procedure [10].

the XUV-IR delay an ideal tool for measuring the relative phase of consecutive harmonics [5,12].

In the experiment, argon was used as an ionization target, because of the availability of calculated matrix elements for its mixed-color two-photon ionization [13]. The favorable cross sections for XUV single-photon ionization of Ar facilitate the detection of high-order harmonics. The ionization potential of $\operatorname{Ar}\left(\sim 10 \hbar \omega_{\text {IR }}\right)$ limits detection to harmonics with order 11 and higher. The highest detected order, 21, is limited by the present geometry of the spectrometer.

Typical angle-resolved photoelectron images obtained with harmonics generated in an argon jet are presented in the top part of Fig. 2, where cuts are shown through the $3 \mathrm{D}$ velocity distributions obtained. Prominent rings result from single-photon ionization by the 11th to 21 st harmonic. In the right panel, one can discern sidebands between consecutive rings. The signal associated with these sidebands disappears when the dressing field is blocked.

The wave function of photoelectrons depends on the angle $\theta$ between the laser polarization and the direction of photoelectron escape. From an initially spherical state, the angular dependence comes entirely from the $\cos \theta$ in the dipole operator $\mathbf{E} \cdot \mathbf{r}=E r \cos \theta$. The measured angular distributions of the photoelectrons are the squared norm of this wave function, and contain (for parity reasons) only even powers of $\cos \theta$ up to twice the number of involved photons [14]. For the $n$ th-order sideband, the two-photon signals can thus be expressed in terms of Legendre polynomials $P_{k}$ :
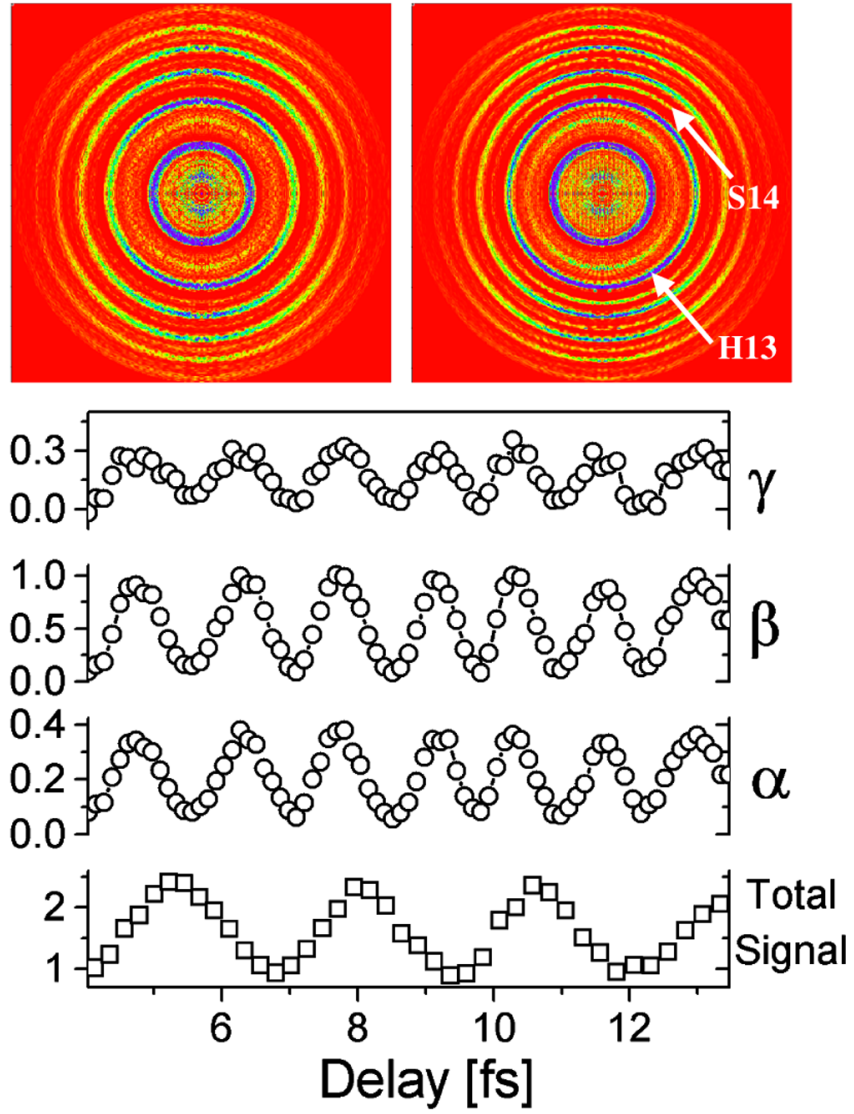

FIG. 2 (color online). (top panels) Slice through the 3D velocity distribution measured for mixed-color (XUV + IR) two-photon ionization of argon, where the XUV light has been obtained by high-harmonic generation in argon. The 3D velocity distribution contains a series of prominent rings resulting from ionization by one XUV photon (11th-21st harmonic) and weaker sidebands at intermediate energies where two mixedcolor ionization processes involving the adjacent harmonics interfere. Arrows in the measurement on the right indicate photoelectron signals due to one-photon ionization with the 13th harmonic (H13) and the sideband resulting from twophoton ionization involving the 13th and the 15th harmonic (S14). In the image on the left, the interference is destructive for most sidebands, whereas in the image on the right it is constructive. (bottom panel) Extracted parameters $\alpha, \beta$, and $\gamma$ [defined in Eq. (1)] for sideband $S 16$ that contains interfering contributions from ionization by the 15 th and 17 th harmonic, as a function of the time delay between the XUV pulse and the IR dressing beam. Both parameter $\alpha$, describing the intensity of the sideband, and parameters $\beta$ and $\gamma$, describing the angular distribution of the ejected photoelectrons in the sideband, oscillate as a function of the delay with half the IR period, and allow the extraction of a phase that contains information about the phase shift between the 15th and 17th harmonic. The total signal oscillates with the full IR period.

$$
S(n, \theta)=\alpha+\beta P_{2}(\cos \theta)+\gamma P_{4}(\cos \theta) .
$$

The parameter $\alpha$ determines the magnitude of the signal integrated over all ejection angles, since the higher $P_{k}$ integrate to zero [15]. 
The bottom part of Fig. 2 shows $\alpha, \beta$, and $\gamma$ for sideband $S 16$ arising from interfering ionization channels involving the 15th and the 17th harmonic, together with a curve representing the total photoelectron signal (the sum of all harmonics and sidebands). This total signal oscillates with a frequency that corresponds to the IR field period of $\sim 2.7 \mathrm{fs}$, since the total laser intensity at the harmonic generation point is slightly affected by the weak dressing beam passing through it. This results in oscillations in the total XUV yield as the phase of the dressing IR is scanned, which can be fit to a functional form $\cos \left[\omega_{\mathrm{IR}} \tau\right]$. The total signal thus allows in situ calibration of the delay $\tau$, which (for small rotation angle of the glass plates) is a quadratic function of this angle. The sideband intensity $\alpha$ can then be fitted on this predetermined delay scale. In the sideband signals, characteristic oscillations $\cos \left[2 \omega_{\mathrm{IR}} \tau+\phi_{\alpha}\right]$ appear at twice the frequency of the oscillations in the total photoelectron yield, since the interference involves one channel where an IR photon is emitted and one where an IR photon is absorbed. Even deeper oscillations can be clearly distinguished in the parameters $\beta$ and $\gamma$, giving us the possibility to extract from our data the three phases $\phi_{\alpha}$, $\phi_{\beta}$, and $\phi_{\gamma}$. The fact that the modulation depths and phases of $\alpha, \beta$, and $\gamma$ are not equal points out an essential modulation of the shape of the angular distributions, rather than merely an overall change of the signal strength.

According to the theoretical treatment of the mixedcolor two-photon ionization, the relative phase of neighboring harmonics can be obtained from $\phi_{\alpha}$ by subtracting a small phase correction $\vartheta_{\alpha}(\sim 0.2 \mathrm{rad}$, dependent on the sideband order) contributed by the atomic response $[5,13]$. Results for a series of experiments using argon, krypton, and xenon as harmonic generation gas are shown in the top panel of Fig. 3. Combining these phase measurements with a measurement of the relative amplitudes of the harmonics determined from the photoelectron spectra, a reconstruction of the XUV temporal shape becomes possible. As shown in the bottom panel of Fig. 3, the reconstructed XUV burst can be as short as $\sim 250 \pm$ 30 as (FWHM) for harmonic orders 11 through 21 when argon is used for high-harmonic generation.

The phase corrections $\vartheta_{\beta}$ and $\vartheta_{\gamma}$ depend only weakly on the harmonic amplitudes, and thus are practically independent of the generation process. As such, they provide a good check on the various atomic phases obtained by theoretical modeling of the ionization process. Results obtained for $\Delta(\alpha \beta)=\phi_{\alpha}-\phi_{\beta}$ are presented in Fig. 4. Within the accuracy of the measurements, all experimental curves coincide. The theoretical curve [12] reproduces the trend in the experimental data quite well, albeit with a slight offset. The theoretical results for $\Delta(\alpha \beta)$ and $\Delta(\beta \gamma)$ remain almost constant when the ratio of the amplitudes of neighboring harmonics is changed in the range between 0.5 and 2 . We can conclude that reconstruction of the XUV pulse shape can be performed both
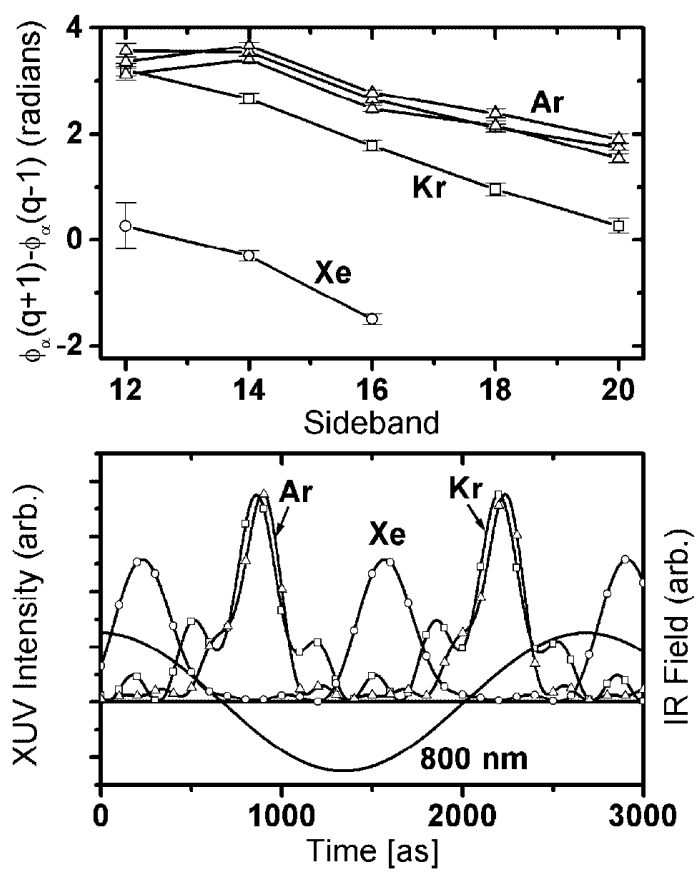

FIG. 3. (top panel) Extracted phase differences $\varphi_{\alpha}(q+1)-$ $\varphi_{\alpha}(q-1)$ between adjacent harmonics for three experiments performed using argon as a harmonic generation gas (open triangles), and using xenon (open circles) and krypton (open squares) as harmonic generation gases. The three argon data sets were taken on different days under conditions as similar as we could make them. (bottom panel) XUV temporal shape for argon, krypton, and xenon as harmonic generation gases, on the basis of the harmonic phase differences shown in the top plot. The harmonic amplitudes needed for the reconstruction were obtained from photoelectron spectra recorded in the absence of the IR dressing beam.

on the basis of sideband intensities and on the basis of the angular distribution measurements.

The reconstruction of the XUV temporal shape also yields a determination of the timing of the XUV bursts with respect to the field oscillations of the femtosecond IR laser. According to the description of high-harmonic generation in terms of a three-step recollision model, the time corresponding to the maximum kinetic energy of recollision of the ionized electrons with their parent ions occurs at a delay of $70 \%$ of the IR period $\left(T_{\mathrm{IR}}\right)$ with respect to the appearance of a field maximum in the IR field $[16,17]$. Taking into account the Gouy phase shift accumulated by the XUV and IR dressing beams between the high-harmonic generation point and the spectrometer [18], we obtain a pulse timing of $(0.83 \pm 0.05) \times T_{\mathrm{IR}}$ for argon and krypton and $(0.59 \pm 0.07) \times T_{\mathrm{IR}}$ for xenon (Fig. 3). This striking difference between argon and krypton versus xenon is most likely due to the fact that in the former case the high-harmonic generation takes place in the tunneling regime, whereas in the latter it takes place in the multiphoton ionization regime. In the latter case, resonant effects can strongly affect recollision times [19]. The observed differences between argon, krypton, and 


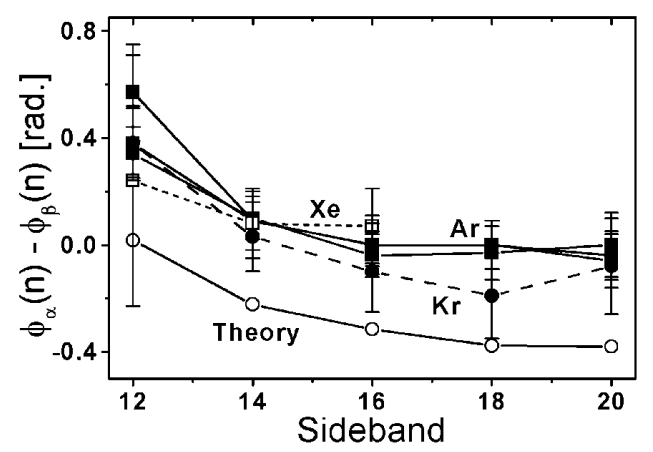

FIG. 4. Difference between the extracted phases $\phi_{\alpha}$ and $\phi_{\beta}$ for three experiments using argon (solid squares), krypton (solid circles), and xenon (open squares) as harmonic generation gas, along with a theoretical prediction based on the mixed-color two-photon ionization model (open circles). Since the phase difference depends only weakly on the harmonic amplitudes and is independent of the harmonic phases, the experimental results lie on top of each other, and agree reasonably well with the theoretical prediction.

xenon underscore the remarkable robustness of the results that have been obtained for these three gases over the course of extensive experiments, where variations in the laser intensity, beam focusing conditions, and harmonic gas densities might have led to variations in the phases determined [20]. It seems that optimization of the harmonic yield and quality of the 2D photoelectron images has ensured that on a day-to-day basis the experiment was performed under rather similar conditions (see the argon results in Figs. 3 and 4).

The phase dependence of angular distributions might be useful for pulse reconstructions under conditions where the XUV spectrum is so broad (due to the short pulse duration) that single- and two-photon signals overlap in energy. The various signals would have opposite parity [21], though, causing odd Legendre polynomials to contribute to the angular distributions [22]. This again allows unambiguous separation of the photoelectron signal at any energy into single- and two-photon contributions, the latter allowing the same kind of analysis as we performed above. It has been proposed [23] that even a single attosecond pulse can be fully characterized through this use of angular information in the perturbative limit [24].

We acknowledge the technical assistance from A. Buijserd and R. Kemper, the efforts of H. L. Offerhaus, E. Springate, and L. Spruit during the early stages of the experiments, and discussions with C. Dinu. This work is part of the research program of the "Stichting voor Fundamenteel Onderzoek der Materie (FOM)," which is financially supported by the "Nederlandse organisatie voor Wetenschappelijk Onderzoek (NWO). It is further funded by EC grant No. HPRN2000-00133 (ATTO network).

*Permanent address: J. J. Thomson Physical Laboratory, University of Reading, Whiteknights, Reading RG6 6AF, UK.

[1] A. H. Zewail, Les Prix Nobel (Almqvist \& Wiksell International, Stockholm, 2000).

[2] R.W. Schoenlein et al., Science 254, 412 (1991).

[3] M. Drescher et al., Nature (London) 419, 803 (2002).

[4] N. A. Papadogiannis et al., Phys. Rev. Lett. 83, 4289 (1999).

[5] P. M. Paul et al., Science 292, 1689 (2001).

[6] M. Hentschel et al., Nature (London) 414, 509 (2001).

[7] Gy. Farkas and Cs. Toth, Phys. Lett. A 168, 447 (1992).

[8] S.E. Harris, J. J. Macklin, and T.W. Hansch, Opt. Commun. 100, 487 (1993).

[9] Y. Silberberg, Nature (London) 414, 494 (2001).

[10] M. J. J. Vrakking, Rev. Sci. Instrum. 72, 4084 (2001).

[11] V. Veniard, R. Taieb, and A. Maquet, Phys. Rev. Lett. 74, 4161 (1995).

[12] J. A. Norin et al., Phys. Rev. Lett. 88, 193901 (2002).

[13] E. S. Toma and H. G. Muller, J. Phys. B 35, 3435 (2002).

[14] P. Lambropoulos, Adv. At. Mol. Phys. 12, 87 (1976).

[15] Note that this $\beta$ is not the "asymmetry parameter" (for which the symbol $\beta$ is also often used). In our notation the latter equals $\beta / \alpha$.

[16] P. B. Corkum, Phys. Rev. Lett. 71, 1994 (1993).

[17] P. Saliere et al., Science 292, 902 (2001).

[18] C. Dinu et al., Phys. Rev. Lett. 91, 063901 (2003).

[19] H. G. Muller, Opt. Express 8, 417 (2001).

[20] M. B. Gaarde and K. J. Schafer, Phys. Rev. Lett. 89, 213901 (2002).

[21] H. G. Muller, P. H. Bucksbaum, D.W. Schumacher, and A. Zavriyev, J. Phys. B 23, 2761 (1990).

[22] Another way of saying this is that a short pulse breaks inversion symmetry, which in the presence of a field is only a symmetry when combined with a time shift of half a cycle.

[23] H. G. Muller, Appl. Phys. B 74, s17 (2002).

[24] The attosecond streak camera used in Ref. [6] uses angular information in the nonperturbative regime, which makes complete reconstruction of complex pulses much less straightforward. 